\title{
Review
}

\section{Overcoming Alcohol Misuse: A 28-Day Guide}

Conor Farran

Blackhall Publishing, 2011, £15.00, pb, 320 pp.

ISBN 9781842182130

Conor Farren has succeeded in creating a comprehensive, practical and easily readable text which aims to bring people who misuse alcohol, their family and friends on a day-by-day journey through the first 28 days of rehabilitation. Here is a manual on overcoming alcohol misuse. The book is organised and well structured. It is divided into three sections: general information about alcohol misuse, the causative factors, and effects of alcohol on health and social life; guidance on the process of involving people who misuse alcohol in their own care; and various techniques helpful in the recovery process.

The core diagnostic features, effects of alcohol misuse and the recovery rehabilitation model have been illustrated with several case studies drawn from real patients and the author's clinical experience. Each chapter ends with 'Joe's story' based on different experiences of people as they journey from alcohol misuse into sobriety. The text is not overly burdened by theory.

This book teaches those who misuse alcohol and their families how to identify alcohol-related problems and what the effects of alcohol misuse are. It also gives trainees a thorough understanding of the experiences of patients with alcohol dependence, their highs and lows as they go through the journey of recovery. There are chapters highlighting links of alcohol misuse with comorbid psychiatric illnesses, particularly depression and mood disorders, and the influence of alcohol on treatment and therapies.

Rajan Chawla, specialist registrar (addiction psychiatry), North Hub Substance Misuse Services, Birmingham and Solihull Mental Health NHS Foundation Trust, Birmingham B23 7LA, UK, email: rajan.chawla@nhs.net doi: $10.1192 / p b . b p .112 .037747$ 